\title{
MATERIAL POINT METHOD FOR HYDRO-MECHANICAL PROBLEMS AND ITS APPLICATION TO SEEPAGE FAILURE ANALYSES
}

\author{
K. Abe $^{1}$, K. Soga ${ }^{2}$ and S. Bandara ${ }^{2}$ \\ ${ }^{1}$ Structual Technology Division, Railway Technical Research Institute (kabe@rtri.or.jp) \\ ${ }^{2}$ Department of Engineering, University of Cambridge
}

\begin{abstract}
In this study, a fluid-soil coupled MPM algorithm based on Biot's theory is proposed for solving hydro-mechanical problems of soil including large deformation problems. The proposed algorithm can be computationally efficient for dealing with the large deformation problems since it uses an Eulerian background mesh for solving the equation of motion of soil in a similar manner as the conventional FEM using physical quantities such as effective stresses and pore water pressures introduced from Lagrangian particles which consist of solid and liquid phases. In order to examine the numerical stability and accuracy of the method, simulations of one-dimensional consolidation test were carried out and the results were compared to the analytical solution. The effect of mesh size and particle number per mesh on the accuracy of the proposed algorithm was also discussed. In order to show the capability of the proposed method, an experiment on a large scale levee failure due to seepage was simulated. The computed large displacements and the behavior of the failed levee matched well with those from the experiment. The effect of change of pore water pressures due to seepage to the effective stress paths in the levee was also discussed.
\end{abstract}

Keywords: Hydro-mechanical problem, Seepage, Material Point Method.

\section{INTRODUCTION}

The conventional Finite Element Method (FEM) has a difficulty in solving large deformation problems because excessive mesh distortions require remeshing. To overcome this, several mesh free methods such as the Element Free Galerkin Method (FEGM) (Belytschko et al., 1994), the Smoothed Particle Hydrodynamics (SPH) (Lucy, 1977; Gingold and Managhan, 1977) and the Material Point Method (MPM) (Sulsky et al., 1995) have been proposed. Recently, MPM has gained popularity for problems of large deformed soil because it computes strain increments in a similar manner as the conventional FEM using the elastoplastic constitutive laws such as Mohr-coulomb model. Moreover, the treatment of boundary conditions is easier comparing with other meshfree methods such as SPH and EFGM because the MPM is one of Patrticles In Cell (PIC) method, i.e., which has not only Lagrangian particles but also Eulerian background meshes, and we can easily describe the Dirichlet (first- 
type) boundary consdition by using nodes of the backgound meshes. In this study, a fluid-soil coupled MPM algorithm based on Biot's theory (Biot, 1962) is proposed for solving hydromechanical problems of soil including large deformation problems. The proposed algorithm can be computationally efficient for dealing with the large deformation problems since it uses an Eulerian background mesh for solving the equation of motion on soils in a similar manner as the conventional FEM using physical quantities such as effective stresses and pore water pressures delivered by Lagrangian particles which consist of solid and liquid phases. In order to examine the numerical stability and accuracy of the method, simulations of onedimensional consolidation test were carried out and the results are compared to the analytical solution. The effect of mesh size and particle number per mesh on the accuracy of the proposed algorithm was also discussed. In order to show the capability of the proposed method, an experiment on a large scale levee failure due to seepage (Iseno et al, 2004) was simulated. The computed large displacements and the behavior of the failed levee matched well with those from the experiment. The effect of change of pore water pressures due to seepage to the effective stress paths in the levee was also discussed.

\section{FLUID-SOIL COUPLED MPM}

\subsection{Algorithm}

The process flow of the algorithm is shown in Figure 1. In this study, at a given time $t^{k}$, the analysis domain consists of two layers of particle - solid layer $\left(N_{s}\right.$ number of solid particles positioned at $\left.\mathbf{x}_{s p}^{k}=\left(x_{s p}^{k}, y_{s p}^{k}\right)\right)$ and water layer $\left(N_{w}\right.$ number of water particles positioned at $\mathbf{x}_{w p}^{k}=\left(x_{w p}^{k}, y_{w p}^{k}\right)$ ), as shown in Figure 2. The water layer calculates the pore water pressure distribution derived from the equation of state and the velocities of water particles based on Darcy's law. On the other hand, the solid layer calculates the effective stress, the velocity and the deformation of soil skeleton. The stationary Eulerian computational mesh is composed of nodes $\left(i=1, . . N_{n}\right)$ positioned at $\mathbf{x}_{i}=\left(x_{i}, y_{i}\right)$. At node $i$, the nodal values of solid and water

phases are computed using the nodal interpolation functions at the location of particle $N_{i}\left(\mathbf{x}_{p}^{k}\right)$. One of the disadvantages of the original MPM formulation is that numerical noises are produced when particles cross the cell boundaries of background mesh (Suslky, 1995; Bardenhagen, 2004). It is possible to reduce such noises by using interpolation function proposed by Bardenhagen (2004).

Figure 3 describes the major features of this algorithm. The physical quantities of water layer such as pore water pressures are delivered by the water particles and that of solid layer are delivered by the solid particles. Then, the nodal accelerations of soil, which is solid and water mixture, are introduced at nodes of background meshes by solving equation of motion of soil and introducing physical quantities delivered by the particles through interpolation functions. Using the accelerations of soil, the nodal velocities of soil is updated. Under the assumption that the relative acceleration of water layer to that of solid layer is much smaller than that of solid layer, the nodal velocities of solid layer is almost same as that of soil. So, using the nodal velocities of soil (or solid layer), we can calculate strain increment of solid layer. On the other hand, the volumetric strain increment of water layer, which is 


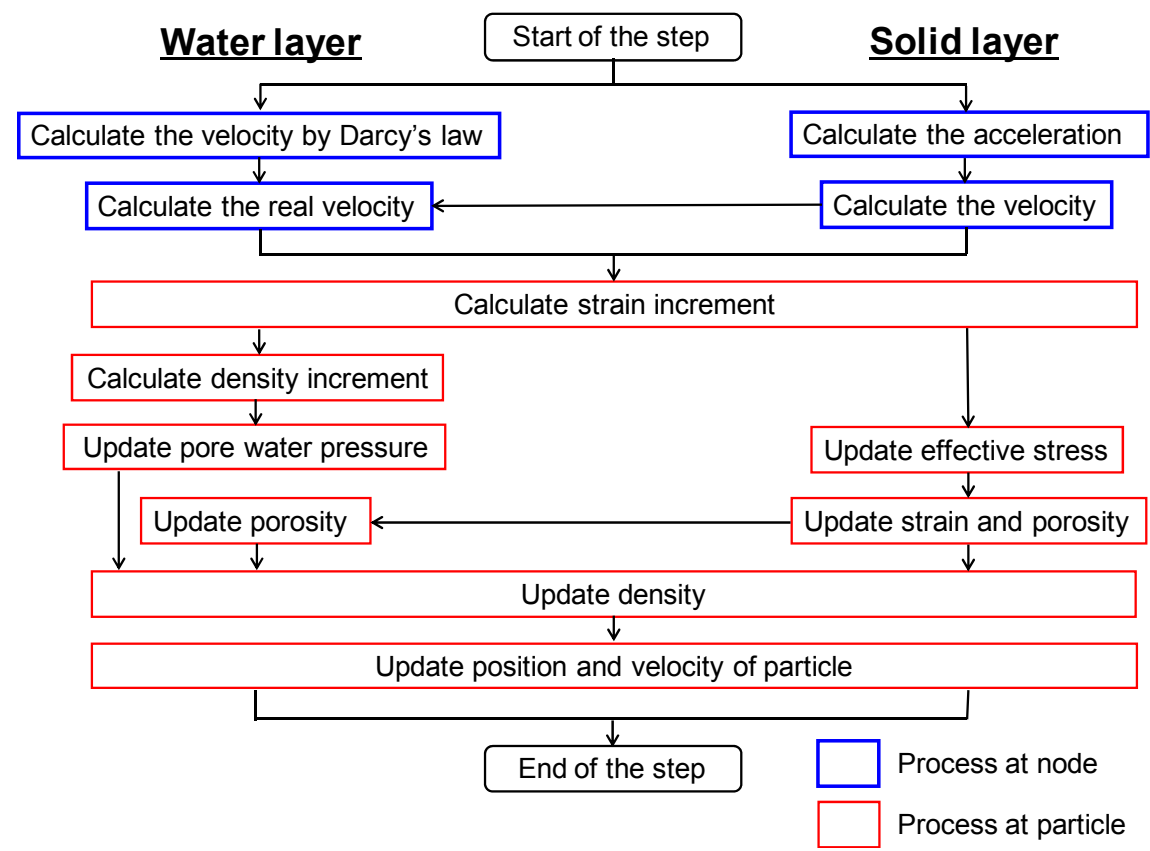

Figure 1. Process flow of the algorithm.

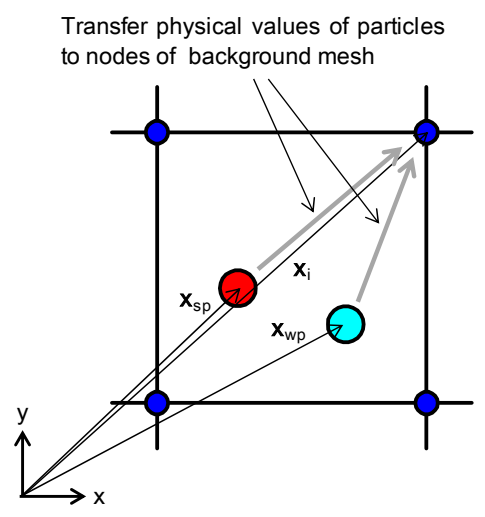

(a) Former stage

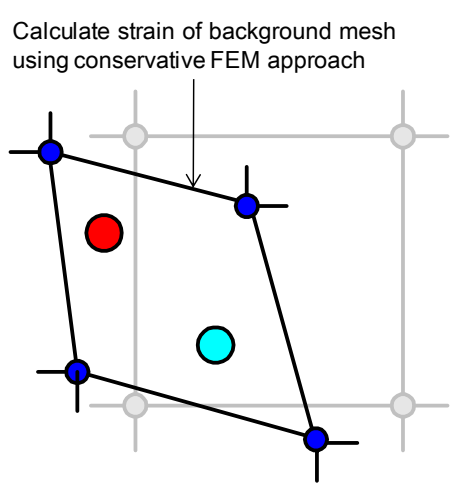

(b) Convention stage
Mesh is reset to the initial configuration, while the particle keeps the position.

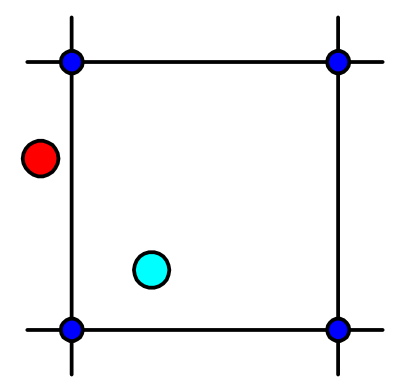

(c) Latter stage

Figure 2. Schematic figure of analysis flow.

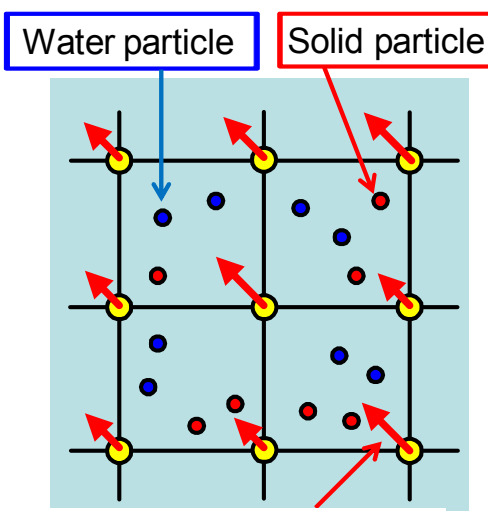

Nodal resultant force of water and solid particles

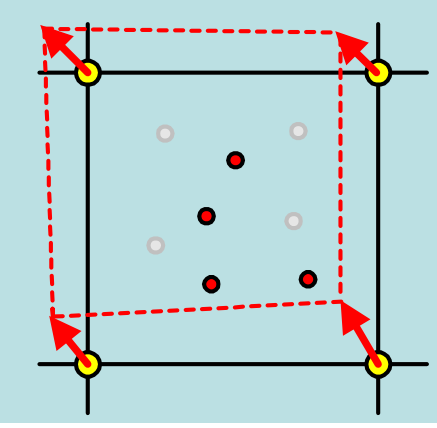

Solid particles

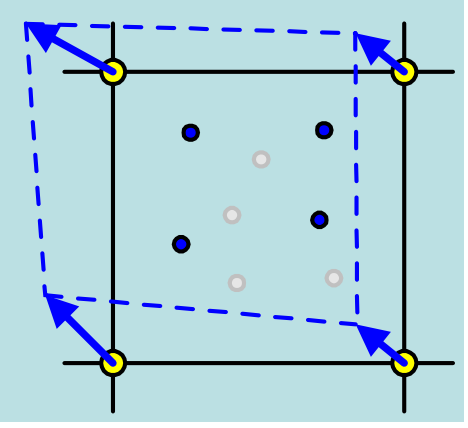

Water particles

Calculate strain increment of solid and water particles.

Figure 3. Major features of the algorithm 
necessary to introduce the pore water pressure, is introduced from the nodal soil-water relative velocities derived by Darcy's law, which are introduced from the following equation:

$$
\mathbf{v}_{w i}^{* k}=k_{s}\left[\sum_{w p=1}^{N_{w}}\left(m_{w p} / \bar{\rho}_{w w p}^{k}\right) \mathbf{G}_{i w p}^{k} \cdot \mathbf{p}_{w p}^{k}-\left\{\sum_{w p=1}^{N_{w}}\left(m_{w p} / n_{w p}^{k}\right) N_{i w p}^{k}\right\}\left(\mathbf{g}-\mathbf{a}_{s i}^{k}\right)\right] / \sum_{w p=1}^{N_{w}} m_{w p} g N_{i_{w p}}^{k}
$$

where, $\mathbf{v}_{w i}^{* k}$ is nodal soil-water relative velocity at node $i$ at time $t^{k}, m_{w p}$ is the mass of water particle $w p$ located at $\mathbf{x}_{w p}^{k}$ at time $t^{k}$, i.e. $m_{w p}=\rho_{w p} V_{w p}\left(V_{w p}\right.$ : the volume of water particle $\left.w p\right)$, $\mathbf{g}$ is the gravitational acceleration, $g$ is the scalar of gravity, $k_{s}$ is the soil permeability, $N_{i w p}^{k}(=$ $\left.N_{i}\left(\mathbf{x}_{w p}^{k}\right)\right)$ is the interpolation function value computed at $\mathbf{x}_{w p}^{k}, \mathbf{G}_{i_{w p}}^{k}=\nabla N_{i w p}^{k}, \mathbf{p}_{w p}^{k}$ is the pore water pressure at $\mathbf{x}_{w p}^{k}$, and $\mathbf{a}_{s i}{ }^{k}$ is the acceleration of solid phase at node $i \cdot \bar{\rho}_{w w p}{ }^{k}$ and $n_{w p}^{k}$ are the averaged density of water phase and the averaged volume fraction of water phase of a control volume at $\mathbf{x}_{w p}^{k}$ at time $t^{k}$, respectively.

The Eq.(1) is introduced as follows. The water velocity of pore fluid based on Darcy's law is governed by Eq. (2):

$$
\mathbf{v}_{w}^{*}=-\frac{k_{s}}{\bar{\rho}_{w} g}\left\{\nabla \mathbf{p}+\frac{\bar{\rho}_{w}}{n}\left(\mathbf{g}-\mathbf{a}_{s}\right)\right\}
$$

where, $\mathbf{a}_{s}$ is the acceleration of solid phase, $n$ is the volume fraction of water phase. $\bar{\rho}_{w}$ is the averaged density of water over total control volume, i.e., $\bar{\rho}_{w}=\rho_{w} n$ ( $\rho_{w}$ is the water density). The following weak form of Eq. (2) is derived as follows:

$$
\int_{\Omega} \bar{\rho}_{w} g \mathbf{v}_{w}^{*} \cdot \mathbf{w} d \Omega=k_{s}\left\{\int_{\Omega} \bar{\rho}_{w} \mathbf{p}^{s}: \nabla \mathbf{w} d \Omega-\int_{\Omega} \frac{\bar{\rho}_{w}}{n}\left(\mathbf{g}-\mathbf{a}_{s}\right) \mathbf{w} d \Omega\right\}
$$

where, $\mathbf{p}^{s}=\mathbf{p} / \bar{\rho}_{w}$ and $\mathbf{w}(\mathbf{x})$ is the test function. In order to obtain Eq. (3), integration by parts and the divergence theorem are applied to the term involving the pore water pressure, as is done for the conventional FEM. The density of water phase at a location $(\mathbf{x})$ at time $t^{k}$ is computed by the sum of water particle masses following the MPM manner:

$$
\bar{\rho}_{w}^{k}(\mathbf{x})=\sum_{w p=1}^{N_{w}} m_{w p} \delta\left\{\mathbf{x}-\mathbf{x}_{w p}^{k}\right\}
$$

where $\delta$ is delta function, and $N_{w}$ is the total numbers of water particles in the background mesh. Substituting Eq. (4) into Eq. (3) results in Eq. (1), which describes nodal soil-water relative velocity $\mathbf{v}_{w_{i}}^{* k}$ at node $i$ at time $t^{k}$.

The pore water pressure increment at the location of water particle $w p\left(\mathbf{x}_{w p}^{k}\right)$ is introduced by Eq. (5) (Klar et al., 2010). This equation is written considering the mass balance of the flow inside pores of a unit control volume of soil: 


$$
\Delta \mathbf{p}_{w p}^{k+1}=\left(K_{w} / \bar{\rho}_{w_{w p}}^{k}\right)\left\{\Delta \bar{\rho}_{w w p}^{* k+1}-\left(\Delta \bar{\rho}_{s w p}^{k+1} / n_{w p}^{k}\right)\right\} \mathbf{I}
$$

where $K_{w}$ is bulk modulus of water. Eq.(5) requires the density changes of water phase $\left(\Delta \bar{\rho}_{w_{w p}}^{* k+1}\right)$ and of solid phase $\left(\Delta \bar{\rho}_{s w p}^{k+1}\right)$ at location $\mathbf{x}_{w p}^{k} \cdot \Delta \bar{\rho}_{w_{w p}}^{* k+1}$ is introduced from the strain increment of water $\Delta \boldsymbol{\varepsilon}_{w_{w p}}^{* k+1}$ by seepage at the location of water particle $w p\left(\mathbf{x}_{w p}^{k}\right)$ using $\mathbf{v}_{w i}^{* k}$ derived from Eq. (1) as follows:

$$
\Delta \boldsymbol{\varepsilon}_{w_{w p}}^{* k+1}=\frac{\Delta t}{2} \sum_{i=1}^{N_{n}}\left\{\mathbf{G}_{i w p}^{k} \mathbf{v}_{w i}^{* k}+\left(\mathbf{G}_{i w p}^{k} \mathbf{v}_{w i}^{* k}\right)^{T}\right\} \quad \text { (6a) } \quad \Delta \bar{\rho}_{w_{w p}}^{* k+1}=-\operatorname{tr}\left(\Delta \boldsymbol{\varepsilon}_{w w p}^{* k+1}\right) \bar{\rho}_{w w p}{ }^{k}
$$

Similarly, $\Delta \bar{\rho}_{s w p}^{k+1}$ is introduced from the strain increment of solid phase at the location of water particle $w p\left(\mathbf{x}_{w p}^{k}\right)$, i.e., $\Delta \mathbf{\varepsilon}_{s_{w p}}^{* k+1}$ using the nodal velocity of solid layer $\mathbf{v}_{s i}^{k+1}$ :

$$
\Delta \boldsymbol{\varepsilon}_{s w p}^{k+1}=\frac{\Delta t}{2} \sum_{i=1}^{N_{n}}\left\{\mathbf{G}_{i w p}^{k} \mathbf{v}_{s i}^{k+1}+\left(\mathbf{G}_{i w p}^{k} \mathbf{v}_{s i}^{k+1}\right)^{T}\right\} \quad \text { (7a) } \quad \Delta \bar{\rho}_{s w p}^{k+1}=-\operatorname{tr}\left(\Delta \boldsymbol{\varepsilon}_{s w p}^{k+1}\right) \bar{\rho}_{w w p}^{k}
$$

Using the updated pore water pressures of water particles and effective stresses of solid particles, which is introduced from constitutive law, the equation of motion of soil is solved at nodes of background meshes, and then the nodal velocities of solid and water layer are updated. Moreover, the velocities, positions, strains, dencities and porocities of solid and water particles are also updated.

\subsection{Stabilization technique}

When coupling analysis is performed with FEM, the full integration with Gauss points may cause numerical instability because the pore water pressure can be evaluated at the different points from the location where the effective stresses are evaluated (Shiomi, 2004). In the proposed method, the effective stresses and pore water pressures are evaluated at the solid and water particles, respectively. Hence, the proposed method can also have instability problems because they are located at different locations which are the Gauss points in the MPM. In order to minimize instability, the pore water pressures are evaluated at the center of the stationary Eulerian computational mesh by replacing $N_{i}\left(\mathbf{x}_{w p}^{k}\right)$ to $N_{i}\left(\mathbf{x}_{w c}^{k}\right)$, where $\mathbf{x}_{w c}^{k}$ is the coordinates at the center of a mesh. This operation also gives us that the grid-crossing errors can be avoided. The effective stresses are also evaluated at the center of the mesh by applying an integration scheme similar to the reduced integration in FEM. This can be done by replacing $\mathbf{G}_{i s p}^{k}\left\{=\nabla N_{i}\left(\mathbf{x}_{s p}^{k}\right)\right\}$ to $\mathbf{G}_{i s c}^{k}\left\{=\nabla N_{i}\left(\mathbf{x}_{s c}^{k}\right)\right\}$, where $\mathbf{x}_{s c}^{k}$ is the coordinates at the center of the mesh. 


\section{NUMERICAL STABILITY AND ACCURACY OF THE METHOD}

A series of MPM simulation of one dimensional consolidation test was performed to examine the accuracy of the proposed formulation for soil-fluid coupled analysis. Soil is assumed to behave as an isotropic linear elastic material. The computed spatial distribution of pore water pressure with time, and the relationship between the average degree of consolidation and the normalized time factor are compared to the analytical solution (Terzaghi and Peck, 1976). The MPM background meshes are shown in Figure 4a. The size of the background mesh is $0.02 \mathrm{~m} \times 0.02 \mathrm{~m}$. The height and width of the soil column are $1.0 \mathrm{~m}$ and $0.1 \mathrm{~m}$, respectively. The total number of particles is 1000 for each phase. Each mesh has 4 particles at the start of the analysis. The time increment is set to be $\Delta t=1.0 \times 10^{-7} \mathrm{sec}$. The soil parameters used for the simulation are listed in Table 1 . A surcharge stress of $10 \mathrm{kPa}$ is applied on the soil particle surface and no gravity force is applied. The side boundaries only allow the soil particles along the boundaries to move in the vertical direction. The water particles placed along the upper, lower and the side boundaries are initially set as undrained condition until the initial pore pressure throughout the soil column is close to $10 \mathrm{kPa}$. The undrained condition is applied by setting the nodal water velocities normal to the boundary to be zero. The upper boundary is then changed to drained condition to initiate consolidation. The computed profiles of pore water pressure distribution at different time factors are compared to the analytical solution in Figure $4 \mathrm{~b}$, whereas the computed consolidation curve (the average degree of consolidation versus the time factor) is compared to the analytical solution in Figure 4c. The average degree of consolidation is evaluated as the ratio of

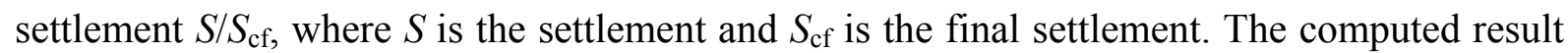
matches well to the analytical solution.

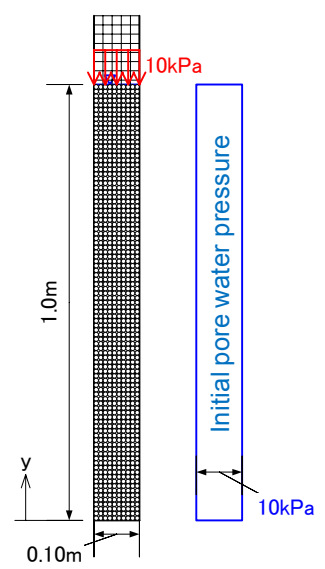

(a) MPM model

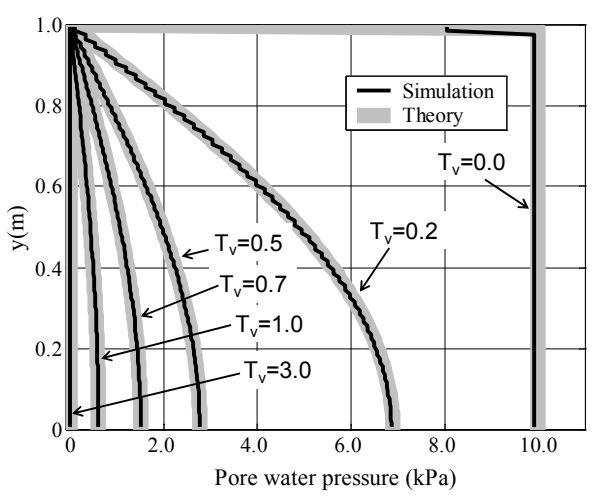

(b) Pore water pressure distribution

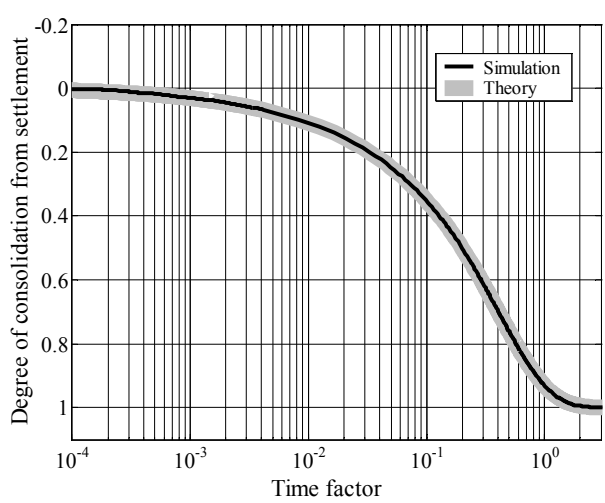

(c) Consolidation curve

Figure 4. Model and result from MPM simulation of one dimensional consolidation test 
Table 1. Parameters of the consolidation simulation.

\begin{tabular}{l|l}
\hline Density of soil $\left(\mathrm{kg} / \mathrm{m}^{3}\right)$ & $1.8 \times 10^{3}$ \\
Density of water $\left(\mathrm{kg} / \mathrm{m}^{3}\right)$ & $1.0 \times 10^{3}$ \\
Young's modulus $(\mathrm{kPa})$ & $2.0 \times 10^{4}$ \\
Poisson ratio & 0.30 \\
Permeability $(\mathrm{m} / \mathrm{s})$ & $5.0 \times 10^{-4}$ \\
Bulk modulus of water $(\mathrm{kPa})$ & $2.2 \times 10^{6}$ \\
Initial porosity of soil & 0.30 \\
Mesh size $(\mathrm{m})$ & 0.02 \\
Time increment $(\mathrm{s})$ & $1.0 \times 10^{-7}$ \\
Number of particles & 2000 (solid: 1000, fluid: 1000$)$ \\
\hline
\end{tabular}

The stability and accuracy of MPM depends on time increment, mesh size and the initial number of particles within each mesh. The number of particles was also varied by changing the initial number of particles at each mesh to be 1, 4 or 9 . The mesh size was varied between $0.01 \mathrm{~m}$ and $0.10 \mathrm{~m}$, which resulted in the number of particles varying between 20 and 18,000. The relative error was evaluated by difference between computed degree of consolidation to the analytical solution. As a result, the error became smaller as the time factor increases and the mesh size decreases and the errors are negligible after $T_{v}=0.1$. We checked that the relationship between the maximum error and mesh size for different numbers of particles in each mesh. Consequently, we found that the error decreases with increasing number of particles in each mesh in most case. However, an opposite trend was observed for the fine mesh case of $L=0.01 \mathrm{~m}$. If the mesh is too fine and the number of particles is too large, the probability of particles crossing the boundary of the background mesh increases and hence more noise is produced. Hence, in order to conduct a successful MPM analysis, a careful selection of mesh size and the number of particles is necessary to increase the numerical accuracy and to reduce the noise especially when time factor $T_{v}$ is less than 0.1 .

\section{APPLICATION TO SEEPAGE FAILURE ANALYSES}

We did simualtion of a large scale physical model experiment of levee failure by seepage carried out by Iseno et al. (2004) and Mori (2008). The schematics of the sandy soil levee model are shown in Figure 5. The model was constructed by soil properties shown in Table 2. A half-section model was used for the experiment and it had a $3.0 \mathrm{~m} \mathrm{high,} 9.0 \mathrm{~m}$ wide, 1:2 slope and $3 \mathrm{~m}$ wide crest. The model was placed on a $20 \mathrm{~cm}$ thick layer of impermeable volume ash clay and the sides were supported by concrete walls. Initially, the model was not saturated. Using a large water tank, a static water pressure was applied at the back of the model, providing seepage flow into the model. The water table in the water tank was kept at $2.3 \mathrm{~m}$ from the bottom of the model. The model showed a small failure at the toe after 12 hours 30 minutes. At 13 hours, the model started to show a progressive failure in 10 minutes and then rapid failure took place for about 30 seconds. The groundwater profile at 6 and 13 hours (wet line) measured by the pore pressure transducers placed inside the model is 
shown in Figure 5. The water profile at 6 hours was used as the initial condition of the simulations. The schematic diagram of the simulation model at the initial condition is shown in Figure 6. The parameters of the simulation are listed in Table 3. The effective stress-strain relation was modeled as the elasto-plastic Mohr-Coulomb model. The base of model was modeled using Coulomb's friction criterion with a basal friction angle of 8.5 degrees. The simulation was carried out by feeding water particles from the left hand side of the levee model to describe the seepage flow from the water tank. The seepage flow in the levee model at 6 hours was modeled by assigning the $x$-component of the initial nodal water velocity of the water particles to be $v_{x w i}{ }^{0}=2.0 \times 10^{-5}(\mathrm{~m} / \mathrm{s})$. Actually, the model had an effect of rainfalls by a rain simulater. However, we neglected that because the effect was not so much comparing to the effect of the seepage flow from the water tank.

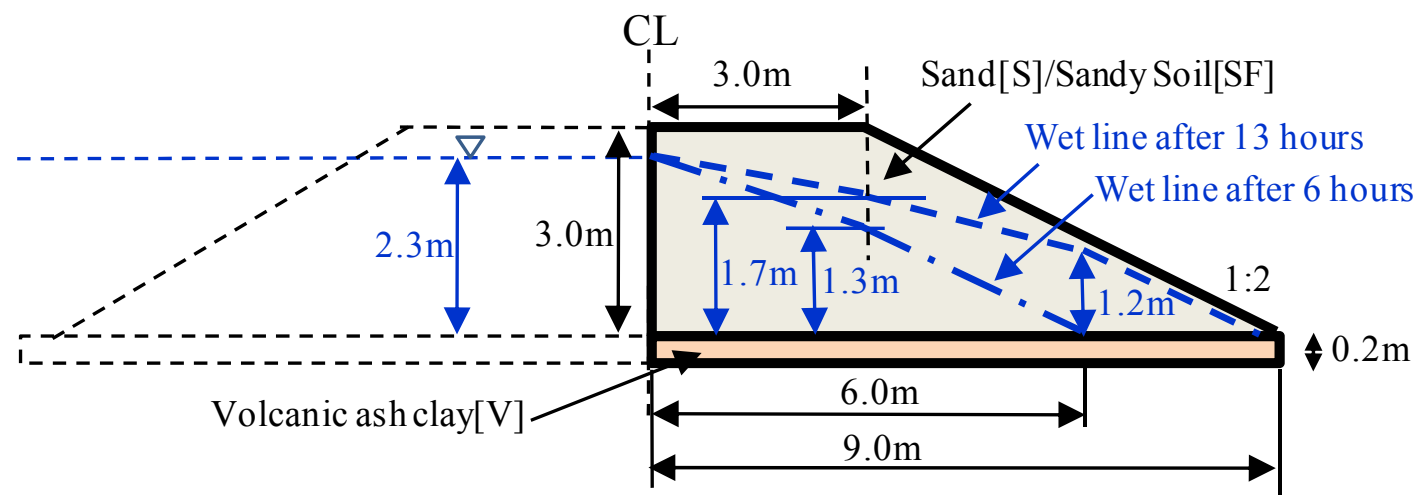

Figure 5. Schematic figure of the experiment model

Table 2. Properties of the sandy soil used in the experiment (after Iseno, 2004; Mori, 2008).

\begin{tabular}{ll}
\hline Soil type & Fine fraction mixed sandy soil \\
Fine fraction content $(\%)$ & 13.0 \\
Grain density $\left(\mathrm{g} / \mathrm{cm}^{3}\right)$ & 2.69 \\
Initial water content $(\%)$ & 19.4 \\
Average degree of compaction $(\%)$ & 75.0 \\
Permeability $(\mathrm{m} / \mathrm{s})$ & $4.5 \times 10^{-5}$ \\
\hline
\end{tabular}

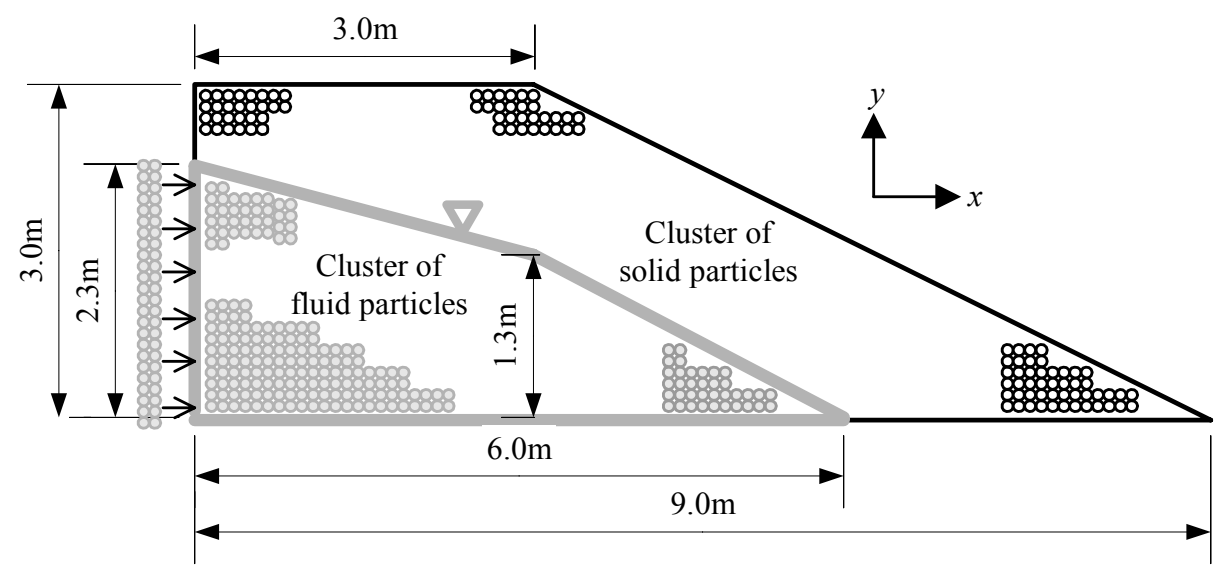

Figure 6. Schematic figure of the simulation model 
Table 3. Parameters of levee failure simulation.

\begin{tabular}{l|l}
\hline Density of grain $\left(\mathrm{kg} / \mathrm{m}^{3}\right)$ & $2.7 \times 10^{3}$ \\
Density of water $\left(\mathrm{kg} / \mathrm{m}^{3}\right)$ & $1.0 \times 10^{3}$ \\
Young's modulus $(\mathrm{kPa})$ & $2.0 \times 10^{4}$ \\
Poisson ratio & 0.30 \\
Cohesion(kPa) & 0.50 \\
Internal friction angle $(\mathrm{deg})$ & 30.0 \\
Dilation angle $(\mathrm{deg})$ & 0.0 \\
Permeability(m/s) & $4.5 \times 10^{-5}$ \\
Bulk modulus of water(kPa) & $2.2 \times 10^{6}$ \\
Initial porosity & 0.30 \\
Basal friction angle(deg) & 8.50 \\
Mesh size(m) & 0.40 \\
Time increment(s) & $1.0 \times 10^{-4}$ \\
Number of particles & 1,475 (solid:935, fluid:540) \\
\hline
\end{tabular}

The results from the simulation are shown in Figure 7, which shows the positions of the particles over time as well as the distribution of deviator strain and pore water pressure in the model. The shape of the levee model is kept as initial one until $8.4 \mathrm{hrs}$, and then the model starts to change its shape, leading to a progressive failure at $8.66 \mathrm{hrs}$ as seepage progresses. The final shape at $8.68 \mathrm{hr}$ is nearly consistent with that from the experiment as shown by the gray line in Figure 7a. The locations of the computed water front line at different times shown in Figure 7a also correspond well to those of the experiment as shown in Figure 5. Figure 7b shows that large deviator strains occur throughout the failing body. Figure $7 \mathrm{c}$ shows that the failure occurred when the pore water pressure reached the toe of the slope.

The effective stress paths are examined at selected points $S_{i}$ in the model as shown in Figure 8. Those are shown in Figure 9. all the effective paths approach the shear failure line, and the soil elements are failing by the increase in pore pressure as well as the decrease in confining stress due to change in slope geometry. This leads to progressive failure of the slope, which was observed both in the experiment as well as in the numerical simulation. This shows the usefulness of the large deformation analysis by MPM to understand the failure mechanism of the levee model. On the other hand, currently, we do not consider the effect of undrained conditions and rainfalls on the behaviour of the levee. This will be included in the levee model in the future. 

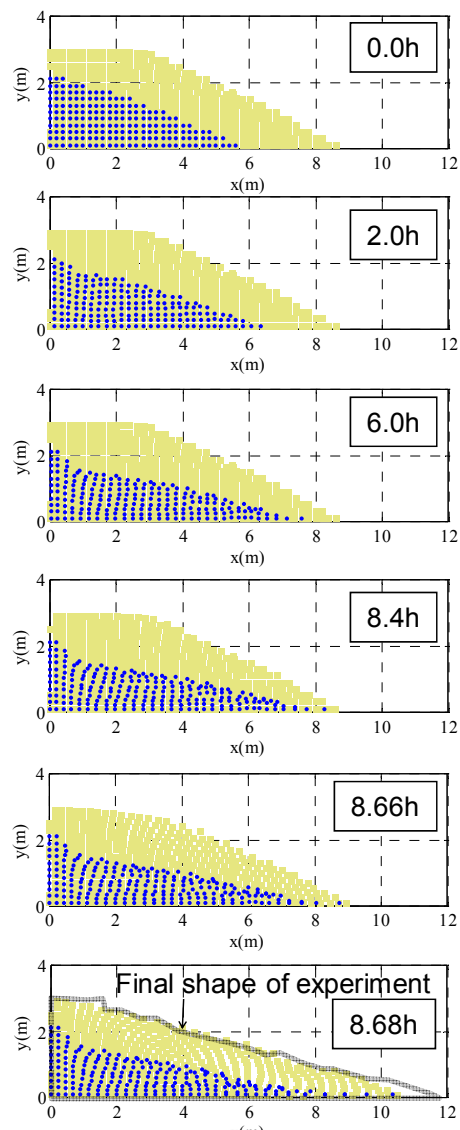

(a) Potions of particles
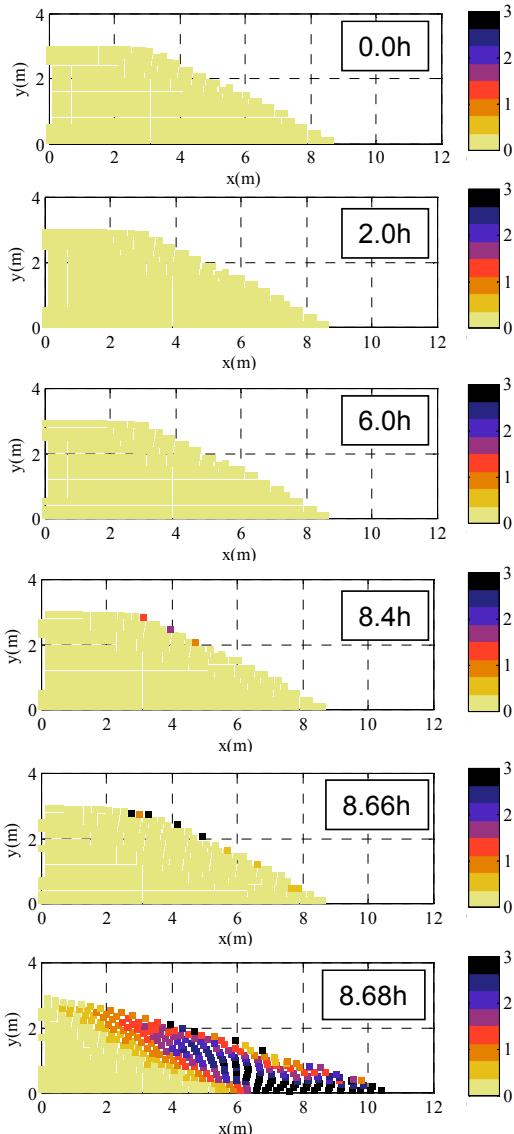

(b) Deviator strain
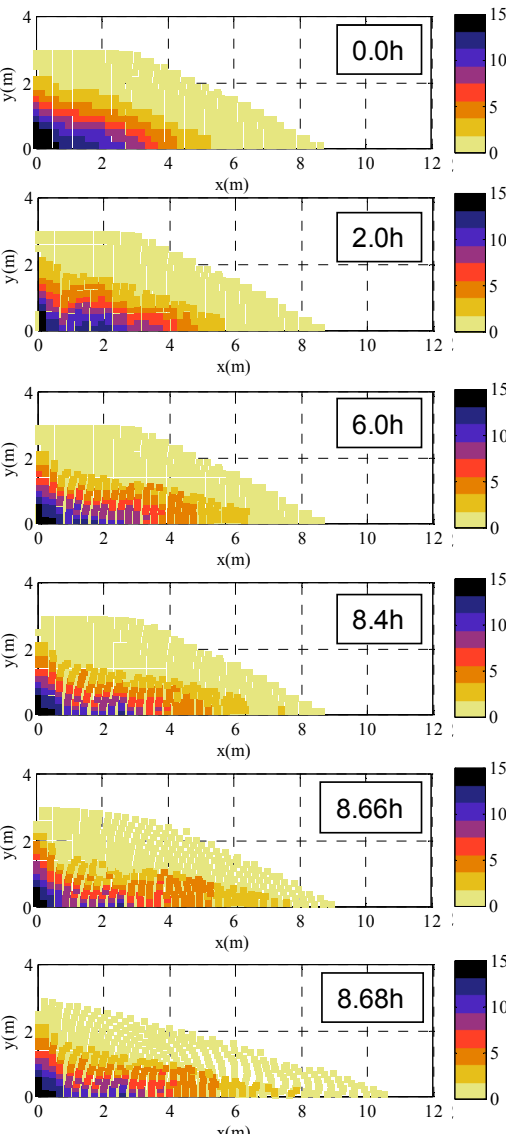

(c) Pore water pressure

Figure 7. Change of considitions of the levee model in simulation

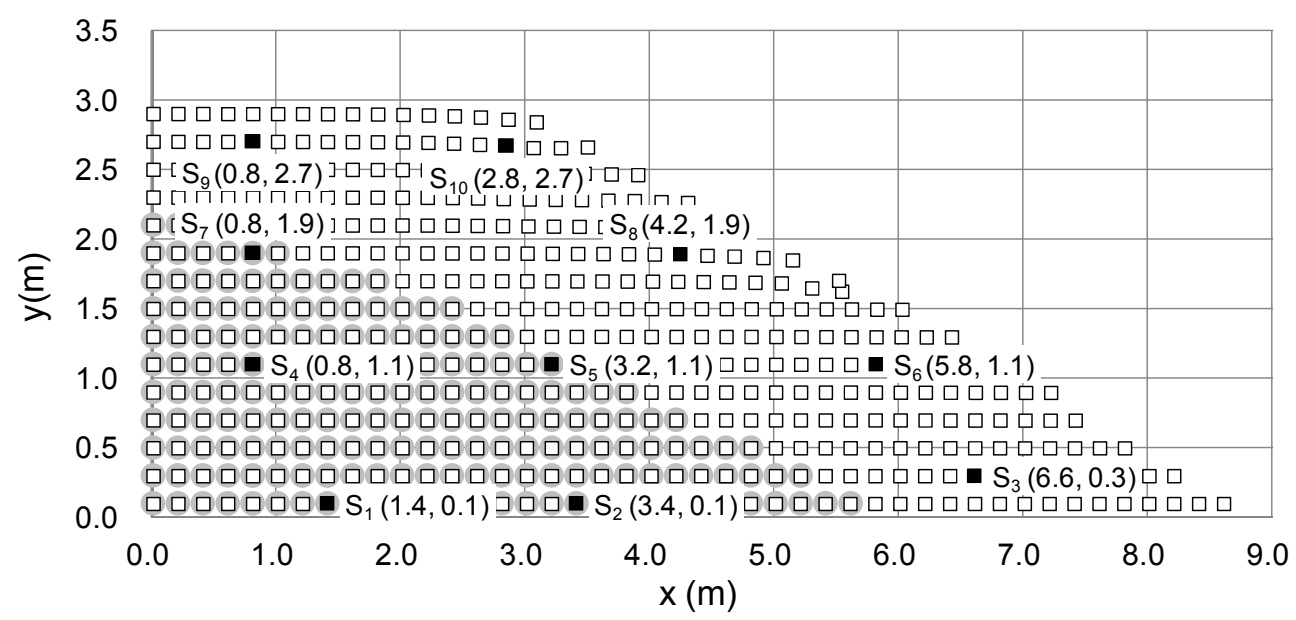

Figure 8. Positions of observation points of effective stress paths at initial state 


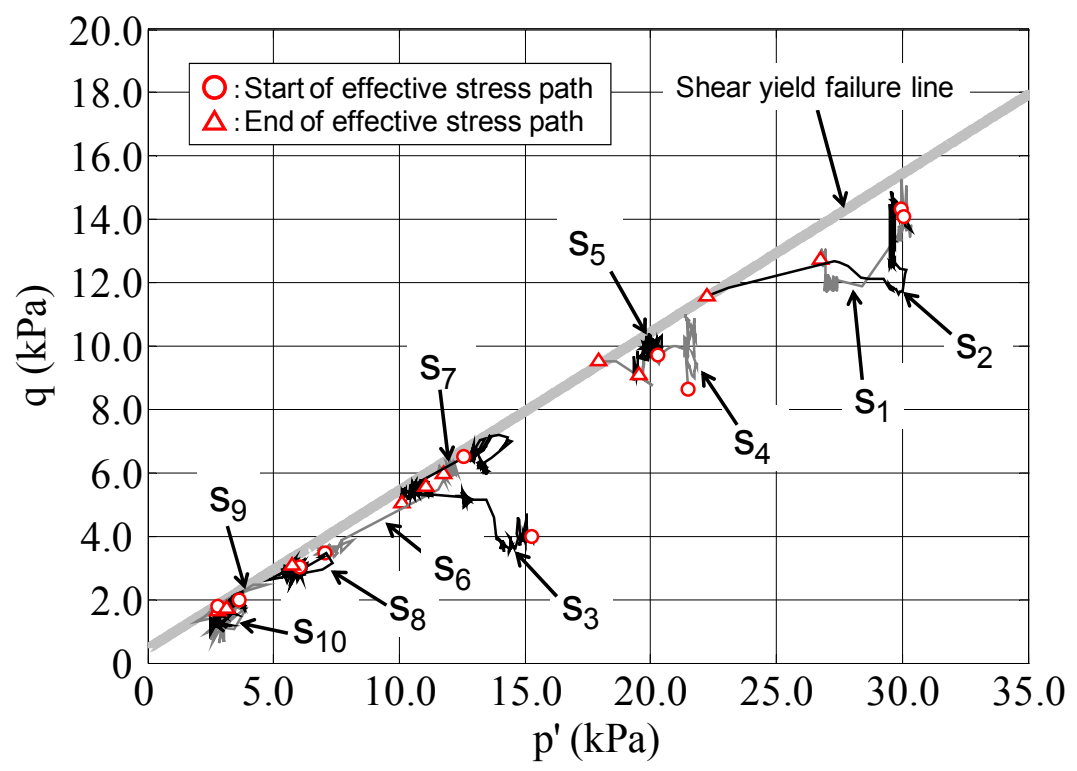

Figure 9. Effective stress paths

\section{CONCLUTIONS}

This study describes a MPM formulation for solving coupled hydro-mechanical problems that involve large deformation of saturated soil. Simulations of one dimensional consolidation test of an elastic soil and the comparison of the result to the analytical solution allowed the examination of the accuracy of the proposed method. The results indicated that careful selection of mesh size and particle density was necessary to obtain accurate results. A large scale levee failure experiment was simulated by using the proposed method. The results were satisfactory in simulating the large deformation observed after rapid failure of the levee due to seepage and the migration of water inside the model. The effect of undrained conditions and rainfalls on the behaviour of soil will be included in the model in the future.

\section{Acknowledgements}

The authors wish to thank Dr. Hirotoshi Mori of Public Works Research Institute (PWRI) in Japan for his useful suggestions and supports on the research work and the sufficient information about the levee failure experiment.

\section{REFERENCES}

[1] Bardenhagen, S.G. and Kober, E.M., "The generalized interpolation material point method" Computer Modeling in Engineering and Science, Vol. 5, No.6, 477-495, 2004.

[2] Belytschko T., Lu Y.Y. and Gu, L., "Element free Galerkin methods," International Journal for Numerical Methods in Engineering, Vol. 37, 229-256, 1994. 
[3] Biot, M.A., "Mechanics of deformation and acoustic propagation in porous media," Journal of Applied Physics, Vol. 33(4), 1482-1498, 1962.

[4] Gingold R.A. and Monaghan J.J., "Smoothed particle hydrodynamics: theory and applications to non-spherical stars," Monthly Notices of the Royal Astronomical Society, Vol. 181, 375-389, 1977.

[5] Iseno, H., Kohashi, H., Furumoto, K., Mori, H. and Ohno, M., "Large model tests of levee reinforcement method with toe drain for seepage failure," Proceedings of Annual symposium of Japanese geotechnical society, Vol. 39, 1255-1256, 2005. (in Japanese)

[6] Klar, A., Soga, K. and Ng, M.Y.A., "Coupled Deformation-Flow Analysis for Methane Hydrate Extraction”, Geotechnique, Vol. 60, No. 10, 765-776, 2010.

[7] Lucy L., "A numerical approach to testing the fission hypothesis," Astronomical Journal, Vol. 81, 1013-1024, 1977.

[8] Mori, H., "The SPH method to simulate river levee failures," Master thesis, University of Cambridge, 2008.

[9] Shiomi, T., "Considerations for instability and accuracy of u-p formulation," Computational mechanics, WCCM VI in conjunction with APCOM '04, Beijing, No.410, 2004.

[10] Sulsky, D., Zhou, S.J. and Schreyer, H. L., "Application of a particle-in-cell method to solid mechanics," Computer Physics Communications, Vol. 87, 236-252, 1995.

[11] Terzaghi K and Peck RB., "Soil Mechanics in Engineering Practice (2nd edn)," Wiley: New York, 1976. 